To be published in Applied Physics Letters

\title{
CONTAMINATION DUE TO MEMORY EFFECTS IN FILTERED VACUUM ARC PLASMA DEPOSITION SYSTEMS*
}

\author{
D.R. Martins ${ }^{1}$, M.C. Salvadori ${ }^{1}$, P. Verdonck ${ }^{2}$ and I.G. Brown ${ }^{3}$ \\ ${ }^{1}$ Instituto de Fisica, Universidade de Sao Paulo, C.P.66318, CEP 05315-970 Sao Paulo, SP, \\ Brazil \\ 2 Laboratory of Integrated System, Ecole Polytechnique of the University of Sao Paulo, \\ CEP 05508-900 Sao Paulo, SP, Brazil \\ ${ }^{3}$ Lawrence Berkeley National Laboratory, 1 Cyclotron Road, Berkeley CA 94720
}

July 2002

* This work was supported by the U.S. Department of Energy under Contract Number DE-AC03-76SF00098. 


\title{
Contamination due to Memory Effects in Filtered Vacuum Arc Plasma Deposition Systems
}

\author{
D.R. Martins and M.C. Salvadori ${ }^{\mathrm{a})}$ \\ Instituto de Fisica, Universidade de Sao Paulo, C.P.66318, CEP 05315-970 Sao Paulo, SP, \\ Brazil \\ P. Verdonck \\ Laboratory of Integrated System, Ecole Polytechnique of the University of Sao Paulo, \\ CEP 05508-900 Sao Paulo, SP, Brazil \\ I.G. Brown \\ Lawrence Berkeley National Laboratory, 1 Cyclotron Road, Berkeley CA 94720
}

\begin{abstract}
$\underline{\text { Abstract }}$
Thin film synthesis by filtered vacuum arc plasma deposition is a widely used technique with a number of important emerging technological applications. A characteristic feature of the method is that during the deposition process not only is the substrate coated by the plasma, but the plasma gun itself and the magnetic field coil and/or vacuum vessel section constituting the macroparticle filter are also coated to some extent. If then the plasma gun cathode is changed to a new element, there can be a contamination of the subsequent film deposition by sputtering from various parts of the system of the previous coating species. We have experimentally explored this effect and compared our results with theoretical estimates of sputtering from the SRIM (Stopping and Range of Ions in Matter) code. We find film contamination of order $10^{-4}-10^{-3}$, and the memory of the prior history of the deposition hardware can be relatively long-lasting.
\end{abstract}

a) Electronic mail: mcsalvadori@if.usp.br 
Thin films of a wide range of materials can be formed using the dense metal plasma that is created in a vacuum arc (also called cathodic arc) discharge. The plasma is formed from the cathode material, which can be selected according to the film wanted. The cathode can be any solid, electrically conducting metal, or carbon, and thus metal films and diamond-like carbon (DLC) films can be deposited. By adding a background reactive gas, ceramic oxides and nitrides can also be formed. The physics and technology of this means of manufacturing metallurgical coatings and the synthesis of thin films and multilayers of many different kinds has become an active field of research in recent years [1-4]. As well as the dense metal plasma that is produced at the cathode spots, metal droplets and other solid cathode debris are also formed. These solid particulates, called macroparticles, can become embedded in the growing film, resulting in poor quality and inferior performance. The favored method for filtering out macroparticles (and also neutral atomic vapor) from the plasma stream is to use a $90^{\circ}$ solenoidal magnetic duct through which the vacuum arc plasma is passed. While the plasma is more-or-less efficiently guided along the curved magnetic field, the uncharged macroparticles and neutrals are lost from the plasma stream. Magnetic duct macroparticle filters have been studied by a number of workers, and several filter variants have been developed [5-8]. An important feature of this method of thin film synthesis is that since the depositing flux is ionized it can be manipulated by an electric field, and by applying a judiciously chosen substrate bias the ion deposition energy can be optimized [9-12]. This in turn provides a means of tailoring the film structure and morphology, and in the case of DLC the $\mathrm{sp}^{3}: \mathrm{sp}^{2}$ (diamond-bonding to graphitic-bonding ratio) can be optimized at a high of around $85 \%[13]$.

For many applications, film purity is an important consideration. Since the film is formed from plasma created from the vacuum arc cathode material, clearly the purity of the cathode material has direct bearing on the film purity. Another factor that can contribute impurities to 
the film is sputtering by the streaming plasma of material that has been previously deposited onto components of the filtered vacuum arc system. It is usual that the same plasma gun assembly and the same macroparticle filter solenoid are used more-or-less continuously, i.e., the plasma gun and filter are not changed when the cathode material is changed in order to deposit a different film material. Then, material of species A that has been deposited onto the surfaces of the plasma gun itself and onto the inside surfaces of the macroparticle filter solenoid can be sputtered by the plasma formed from the cathode of species B, after the cathode has been changed from species A to species B in order to do a new, subsequent deposition of species B. Some of the unwanted, sputtered species-A material can be deposited onto the substrate, both directly as permitted by the system geometry and by ionization and entrainment within the species-B plasma stream. The film of material B can thus be contaminated by the memory that the deposition system maintains of its previous history. We have carried out some straightforward experiments to quantify the magnitude of this contamination effect due to the device history.

A simplified schematic of the experimental set-up is shown in Figure 1. The plasma gun is fabricated from stainless steel, with a ceramic insulator surrounding the $6.3 \mathrm{~mm}$ diameter cathode, and the $6 \mathrm{~cm}$ diameter solenoid constituting the macroparticle filter is made of 6.3 $\mathrm{mm}$ diameter copper tubing. The filter solenoid is freestanding, with no additional tubular section either inside or outside the solenoid. The plasma gun is operated in a repetitively pulsed mode, with $5 \mathrm{~ms}$ pulses at a rate of 1 pulse per second, for these experiments. The solenoid is connected in series with the plasma gun, and so is driven by the same current and at the same time as the plasma gun arc discharge; for the work described here the arc current was $180 \mathrm{~A}$. The substrate, a small (about $1 \mathrm{~cm}$ dimension) piece of silicon wafer, was located about $5 \mathrm{~cm}$ distant from the solenoid exit, and was grounded for the experiments described here. The vacuum pressure during the depositions was about $3 \times 10^{-6}$ Torr. Our experiments 
consisted of carrying out a deposition using one particular kind of cathode material, and then another deposition onto a new substrate with another kind of cathode material. To change from the first species to the second species, the vacuum system was let up to air and the plasma gun cathode changed; we also changed the ceramic cathode insulator at the same time. That is, memory of the first species by the cathode insulator was removed. For each new deposition, the substrate was prevented from viewing the plasma stream for a certain number of plasma pulses immediately prior to starting the deposition, thus allowing the system a "clean-up period".

To determine what cathode materials to use for the experiments, we used the SRIM program $[14,15]$ to calculate sputtering yields. The incident ion energies used for the sputtering calculations were those reported by Anders and Yushkov [16] for ion drift energies of metal ion species in vacuum arc plasmas. Sputtering yields, $\mathrm{Y}_{\mathrm{AB}}$ (atoms of type $\mathrm{B}$ sputtered by incident ions of type A), were calculated for normal incidence only, and we assume that the ratios between the various $\mathrm{Y}$ values are approximately maintained as the angle of incidence varies. The results of these calculations are shown in Table I. For the purpose of the work described here, we wanted to select cathode materials that when used consecutively result in high contamination levels so as to be readily quantifiable by Rutherford backscattering spectrometry (RBS) and so that our results provide an upper limit to the magnitude of these effects. We chose to use (i) the sequence $\mathrm{V}, \mathrm{Nb}, \mathrm{Ta}, \mathrm{Nb}, \mathrm{V}$, and (ii) Ta followed by $\mathrm{Nb}$.

In the first experiment we measured the magnitude of the contamination. The plasma gun was operated with a vanadium cathode for 540 pulses, after which the cathode was changed to niobium, with the ceramic trigger insulator changed at the same time. The system was pumped down to operating vacuum, and the plasma gun fired for 60 clean-up pulses prior to 
inserting the new substrate. With the fresh substrate in place, 300 pulses of $\mathrm{Nb}$ were fired so as to form a $\mathrm{Nb}$ film on the silicon substrate. The $\mathrm{Nb}$ sample was removed for subsequent RBS analysis so as to quantify the level of contamination of $\mathrm{V}$ in the $\mathrm{Nb}$ film. This cycle was repeated using a tantalum cathode: cathode changed to Ta with new trigger insulator, system pumped down, 60 clean-up pulses fired prior to insertion of a new substrate, 300 pulses of Ta fired to form a $\mathrm{Ta}$ film on $\mathrm{Si}$ substrate, and finally the Ta sample removed for RBS quantification of the $\mathrm{V}$ contamination in the $\mathrm{Ta}$ film. Then the cycle was repeated for a $\mathrm{Nb}$ cathode again, and finally for a $\mathrm{V}$ cathode again. In this way we made four samples, each with expected contamination of the prior cathode material - Nb:V, Ta: $\mathrm{Nb}, \mathrm{Nb}: \mathrm{Ta}$, and $\mathrm{V}: \mathrm{Nb}$, where by A:B we mean that the sample is of metal A with an expected small amount of metal B as contaminant. RBS using $2.4 \mathrm{MeV} \mathrm{He}^{+}$ions was carried out on the samples. The results are shown in Table II. Two conclusions can be drawn from these data: the fractional contamination is of order $10^{-4}$ to $10^{-3}$, and the contamination ratios are consistent with the SRIM-calculated sputtering yields.

In the second experiment we investigated the persistence of the contamination - the duration of the memory of the system's past history. Firstly the plasma gun was operated with a Ta cathode for 300 pulses. Then the cathode and insulator were changed and a $\mathrm{Nb}$ cathode inserted, following which the system was operated with the new $\mathrm{Nb}$ cathode for a variable number of clean-up pulses. After the clean-up process a fresh $\mathrm{Si}$ substrate was positioned near the filter exit (without breaking vacuum) and a $\mathrm{Nb}$ film formed with 300 plasma gun pulses. This cycle was repeated a number of times, with a varying number of clean-up pulses. In this way we made samples for subsequent RBS analysis in which the $\mathrm{Nb}$ film was the same apart from the number of clean-up pulses used to erase the memory of the previous Ta operation. The results are shown in Figure 2. These data indicate that contamination in the deposition of new metal species by a prior metal species is a long-lasting 
effect. The fractional contamination, which is of order $10^{-3}$ here (recall we selected high sputter yield species so as to maximize the effect), decays by only about $10 \%-20 \%$ per 100 pulses. Since typical film depositions in this kind of system rarely call for more than several thousand pulses, the contamination can remain significant throughout the deposition.

The experiments described here show that film contamination in repetitively-pulsed filtered vacuum arc deposition systems due to system memory of prior deposition history can be a concern in applications requiring high film purity. The magnitude of the contaminant can be of order $10^{-4}-10^{-3}$ of the primary film material, and the effect (contaminant injection) can be persistent. One obvious way to alleviate this effect would be to use a dedicated plasma gun and filter solenoid for each metal species employed, thus coating the hardware with the wanted atomic species. In the experiments described here we have not investigated the variation of contamination with operating parameters such as arc current and filter coil magnetic field strength, nor contamination of the film by sources other than previous cathode material.

This work was supported by the CNPq (Conselho Nacional de Desenvolvimento Científico e Tecnológico) and by FAPESP (Fundação de Amparo à Pesquisa do Estado de São Paulo), Brazil. The authors are grateful to the "Laboratorio de Analise de Materiais por Feixes Ionicos" (LAMFI), of the Institute of Physics of the University of São Paulo, for the RBS analysis. 
$\underline{\text { References }}$

1. Vacuum Arc Science and Technology, edited by R.L. Boxman, P.J. Martin and D.M. Sanders (Noyes, New York, 1995).

2. I.G. Brown, Cathodic Arc Deposition of Films, in Annual Review of Material Science (Annual Reviews, Palo Alto, 1998), Vol. 28, p. 243.

3. P.J. Martin and A. Bendavid, Thin Solid Films 394, 1 (2001).

4. A. Anders, Surf. Coat. Technol. 93, 158 (1997).

5. I.I. Aksenov, V.A. Belous, V.G. Padalka and V.M. Khoroshikh, Sov. J. Plasma Phys. 4, 425 (1978).

6. A. Anders, S. Anders and I.G. Brown, Plasma Sources Sci. Technol. 4, 1 (1995).

7. A. Anders, Surf. Coat. Technol. 120-121, 319 (1999).

8. M.M.M. Bilek, Y. Yin and D.R. McKenzie, IEEE Trans. Plasma Sci. 24, 1165 (1996).

9. I.G. Brown, X. Godechot and K.M. Yu, Appl. Phys. Lett. 58, 1392 (1991).

10. A. Anders, S. Anders, I.G. Brown, M.R. Dickinson and R.A. MacGill, J. Vac. Sci. Tech. B 12, 815 (1994).

11. O.R. Monteiro, Z. Wang and I.G. Brown, J. Mater. Res. 12, 2401 (1997).

12. Handbook of Plasma Immersion Ion Implantation and Deposition, edited by A. Anders (Wiley, New York, 2000).

13. G.M. Pharr, D.L. Callahan, S.D. McAdams, T.Y. Tsui, S. Anders, A. Anders, J.W. Ager, I.G. Brown, C.S. Bhatia, S.R.P. Silva and J. Robertson, Appl. Phys. Lett. 68, 779 (1996).

14. J.F. Ziegler and J.P Biersack, "Stopping and Range of Ions in Matter". See the web site Www.SRIM.org

15. J.F. Ziegler, J. Appl. Phys. 85, 1249 (1999).

16. A. Anders and G.Yu Yushkov, J. Appl. Phys. 91, 4824 (2002). 
Table I.

Sputtering yields for a number of different metal ions incident upon targets of the same range of elemental species, for incident ion energies as indicated.

\begin{tabular}{|cc|cccccccccc|}
\hline \multicolumn{2}{|c|}{ Incident ion } & \multicolumn{7}{c|}{ Target atomic species } \\
\hline Species & Energy $(\mathbf{e V})$ & $\mathbf{V}$ & $\mathbf{N i}$ & $\mathbf{C u}$ & $\mathbf{N b}$ & $\mathbf{P d}$ & $\mathbf{A g}$ & $\mathbf{T a}$ & $\mathbf{P t}$ & $\mathbf{A u}$ \\
\hline $\mathbf{V}$ & 70 & 0.06 & 0.24 & 0.35 & 0.15 & 0.42 & 0.48 & 0.20 & 0.063 & 0.42 \\
$\mathbf{N i}$ & 41 & 0.009 & 0.057 & 0.14 & 0.007 & 0.22 & 0.25 & 0.085 & 0.14 & 0.18 \\
$\mathbf{C u}$ & 57 & 0.014 & 0.070 & 0.14 & 0.10 & 0.33 & 0.39 & 0.17 & 0.27 & 0.37 \\
$\mathbf{N b}$ & 128 & 0.035 & 0.12 & 0.21 & 0.090 & 0.52 & 0.63 & 0.36 & 0.63 & 0.87 \\
$\mathbf{P d}$ & 80 & 0.008 & 0.033 & 0.067 & 0.020 & 0.18 & 0.29 & 0.052 & 0.40 & 0.58 \\
$\mathbf{A g}$ & 69 & 0.004 & 0.024 & 0.050 & 0.015 & 0.15 & 0.22 & 0.20 & 0.37 & 0.51 \\
$\mathbf{T a}$ & 136 & 0.007 & 0.030 & 0.070 & 0.012 & 0.12 & 0.18 & 0.14 & 0.36 & 0.59 \\
$\mathbf{P t}$ & 67 & 0.0001 & 0.001 & 0.008 & 0.001 & 0.024 & 0.040 & 0.022 & 0.08 & 0.175 \\
$\mathbf{A u}$ & 49 & 0.0001 & 0.0005 & 0.001 & 0.0001 & 0.007 & 0.015 & 0.008 & 0.042 & 0.088 \\
\hline
\end{tabular}


Table II.

Measured (RBS) contamination levels for several different depositions and calculated (SRIM) sputtering yield ratios.

\begin{tabular}{|cccc|}
\hline Deposition & Film:Contaminant & $\begin{array}{c}\text { Measured contaminant } \\
\text { fraction (a) }\end{array}$ & $\begin{array}{c}\text { Predicted contaminant } \\
\text { fraction (normalized) (b) }\end{array}$ \\
\hline $\mathbf{V}$ & - & - & - \\
$\mathbf{N b}$ & $\mathbf{N b : V}$ & $\left(<10^{-3}\right)$ & $0.8 \times 10^{-4}$ \\
$\mathrm{Ta}$ & $\mathrm{Ta:Nb}$ & $\left(<10^{-3}\right)$ & $0.3 \times 10^{-4}$ \\
$\mathbf{N b}$ & $\mathbf{N b : T a}$ & $(8 \pm 1) \times 10^{-4}$ & $8 \times 10^{-4}$ \\
$\mathbf{V}$ & $\mathbf{V : N b}$ & $(4 \pm 1) \times 10^{-4}$ & $3.3 \times 10^{-4}$ \\
\hline
\end{tabular}

(a) RBS-measured fraction of species $\mathrm{B}$ in film of species $\mathrm{A}$. For the $\mathrm{Nb}$ and $\mathrm{Ta}$ films, the measurement is resolution-limited to about $10^{-3}$ because the contaminant is the heavier species (RBS limitation).

(b) Contaminant fraction predicted from the SRIM-calculated sputtering yields of Table 1 after normalizing to the $\mathrm{Nb}$ :Ta experimental value. 


\section{Figure Captions}

Fig. 1 Simplified schematic of filtered vacuum arc deposition system.

Fig. 2 Decay of contamination of $\mathrm{Ta}$ in a $\mathrm{Nb}$ film as a function of number of clean-up pulses prior to insertion of the substrate into the plasma. 
QuickTime ${ }^{\mathrm{TM}}$ and a

Photo - JPEG decompressor
are needed to see this picture.

Figure 1 
QuickTime ${ }^{\mathrm{TM}}$ and a
Photo - JPEG decompressor
are needed to see this picture.

Figure 2 